\title{
From supergiant stars to galaxies: The path to extragalactic distances
}

\author{
Fabio Bresolin and Rolf-Peter Kudritzki \\ Institute for Astronomy, University of Hawaii, \\ 2680 Woodlawn Drive, 96822 Honolulu, HI, USA \\ email: bresolin@ifa.hawaii.edu, kud@ifa.hawaii.edu
}

\begin{abstract}
The spectroscopic study of blue supergiants in nearby galaxies can yield crucial information about the spatial distribution of extinction by dust, and can be successfully used to measure the metal content, abundance patterns, and distances of galaxies out to $\sim 8 \mathrm{Mpc}$ with current telescopes and instrumentation. We briefly review the results of an ongoing project in which the quantitative analysis of B- and A-type supergiants is used to independently test widely used metallicity diagnostics for star-forming galaxies and derive reddening-free extragalactic distances.
\end{abstract}

Keywords. galaxies: distances and redshifts, stars: early-type, stars: abundances, supergiants, HII regions, galaxies: abundances

\section{Introduction}

The quest for improved extragalactic distances is tied to the search for an everincreasing precision in the determination of the fundamental cosmological parameters, in particular the rate of expansion of the universe, $\mathrm{H}_{0}$. Crucial obstacles to establish $\mathrm{H}_{0}$ with an accuracy of $1-2 \%$, as required for improved constraints on neutrino physics and dark energy (Suyu et al. 2012), are represented by lingering systematic uncertainties plaguing our best primary (Cepheids) and secondary (such as type Ia supernovae and the tip of the red giant branch-TRGB - method) distance indicators. Among these are the ill-understood effects of metallicity on distances obtained from the Cepheid periodluminosity relationship, despite a large body of empirical (e.g., Kennicutt et al. 1998; Macri et al. 2006) and theoretical (see Bono et al. 2008) work, and the effects of patchy interstellar extinction by dust, both of which can introduce systematic errors in distances to individual galaxies of $\sim 10 \%$, as shown from spectroscopy of blue supergiants in nearby galaxies (Kudritzki et al. 2008, 2012).

The task of establishing accurate distances to nearby galaxies is inextricably linked to that of measuring their metallicities, and in particular the chemical composition of stellar distance indicators, such as Cepheids or red giants. However, only rarely are the metallicities of the indicators directly measured. In the vast majority of cases HiI region 'metallicities' (in essence their $\mathrm{O} / \mathrm{H}$ abundance ratios) are used to characterize the metal content of the host galaxies (when dealing with Population I objects, such as Cepheids). Unfortunately, various nebular-abundance diagnostics making use of the emission lines present in His region spectra lead to well-known systematic differences of up to 0.6 dex (Bresolin et al. 2004, 2009; Kewley \& Ellison 2008). Since the investigation of the chemical evolution of star-forming galaxies relies on this kind of emission-line diagnostics, it is 
crucial to establish which of these diagnostics better represents the 'true' metallicity of galaxies.

\section{Blue supergiants as distance indicators}

The derivation of reddening-corrected extragalactic distances and metallicities has greatly benefited from low-resolution, high signal-to-noise ratio ( $>50)$ quantitative spectroscopy of young blue supergiant stars located in nearby (distances $D<8 \mathrm{Mpc}$ ) galaxies, as our group has demonstrated in recent years (Kudritzki et al. 2012; and references therein). Because of their extreme brightness in the optical $\left(-10<M_{V}<-7\right.$ mag), Band A-type supergiants are ideal tools to gather crucial information about the chemical composition (of elements like C, N, O, Mg, Al, S, Si, Ti, Fe, Cr) of the young stellar populations in galaxies, providing important constraints on their star-formation histories and chemical evolution. Moreover, we have shown that a technique developed by Kudritzki et al. (2003, 2008), in which spectroscopically available observables (stellar effective temperature $T_{\text {eff }}$, gravity $g$, metallicity, and extinction) are simultaneously obtained and combined, can yield distances to individual galaxies that are accurate to $\sim 5 \%$. The flux-weighted gravity-luminosity relation (FGLR) introduced and discussed in those papers is a simple, tight correlation between the flux-weighted gravity, $g_{\mathrm{F}}=g / T_{\text {eff }}^{4}$, and the luminosity of B/A supergiants. Such a correlation derives naturally from the characteristics of the post-main-sequence evolution of massive $\left(12<M<40 \mathrm{M}_{\odot}\right)$ stars, which occurs at both constant luminosity $\left(L\right.$ or $\left.M_{\mathrm{bol}}\right)$ and constant mass. It can thus be easily shown (Kudritzki et al. 2003) that a relation of the form $M_{\mathrm{bol}}=a \log \left(g_{\mathrm{F}}\right)+b$ is obtained. Empirical verification of the FGLR has been provided through quantitative spectroscopy of blue supergiants in a number of different galaxies (see Fig. 1).

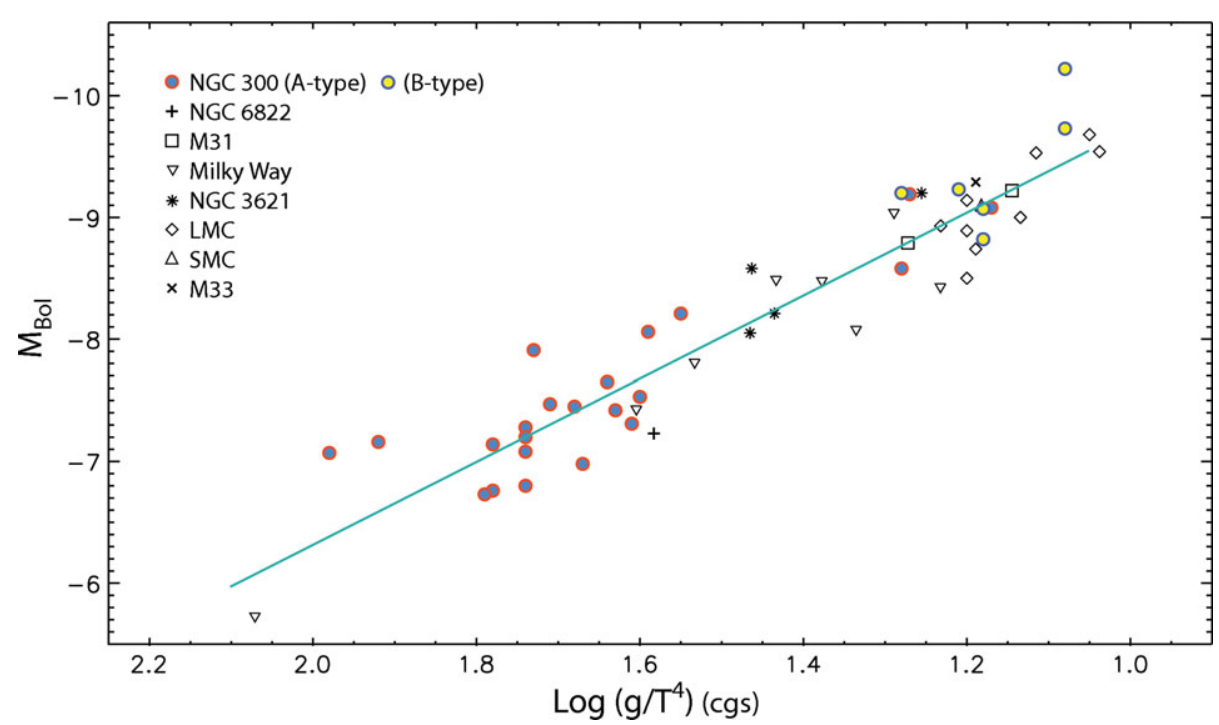

Figure 1. Flux-weighted gravity-luminosity relation for (B- and A-type) blue supergiants in eight galaxies (adapted from Kudritzki et al. 2008).

The advantage of this spectroscopic method is that metallicity and extinction values can be determined individually on an object-by-object basis, thus significantly 
reducing the systematic uncertainties affecting competing methods such as Cepheids or the TRGB, where such an approach is generally not feasible. The patchy distribution of dust in star-forming galaxies is a major concern when optical data are used to derive extragalactic distances. The blue supergiant work has shown that in various cases the reddening analysis of previous work (e.g., Cepheids in the Hubble Space Telescope Key Project on the Extragalactic Distance Scale) could lead to systematic errors on the order of $0.1 \mathrm{mag}$ in $E(B-V)$, which translate into significant errors in the derived distance moduli (see, for a recent review, Kudritzki \& Urbaneja 2012). Although shifting to near-infrared wavelengths largely mitigates this concern, optical photometric studies of extragalactic Cepheids are still being widely carried out, and are thus potentially affected by the difficulties outlined above concerning the reddening estimation.

Our project has been targeting key galaxies for the distance scale (e.g., the maser host galaxy NGC 4258; paper in prep.) or galaxies which are particularly interesting from various scientific perspectives (e.g., NGC 300: Kudritzki et al. 2008; M81: Kudritzki et al. 2012; WLM: Bresolin et al. 2006, Urbaneja et al. 2008; M33: U et al. 2009). The calibration of the FGLR presented in Kudritzki et al. (2008), based on Cepheid distances to eight galaxies, has thus far been used to measure distances as part of our project. A recalibration of the method, based on spectroscopy of blue supergiants in the Large Magellanic Cloud, whose distance can be obtained independently from Cepheids (e.g., using eclipsing binaries: Pietrzynsky et al. 2009) will soon be made available (M. Urbaneja et al., in prep.). As a recent example of the application of the method, we show in Fig. 2 the FGLR of blue supergiants in the spiral galaxy M81, obtained by Kudritzki et al. (2012). Adjustment of the zero point of the local relation, while keeping the slope fixed to that of the calibrating sample, yields a best-fitting distance modulus of $\mu=27.7 \pm 0.1 \mathrm{mag}$. This value compares very well with the TRGB distance of $\mu=27.69 \pm 0.04$ mag by Rizzi et al. (2007) and the Cepheid distance of $\mu=27.80 \pm 0.08$ mag by Freedman et al. (2001).

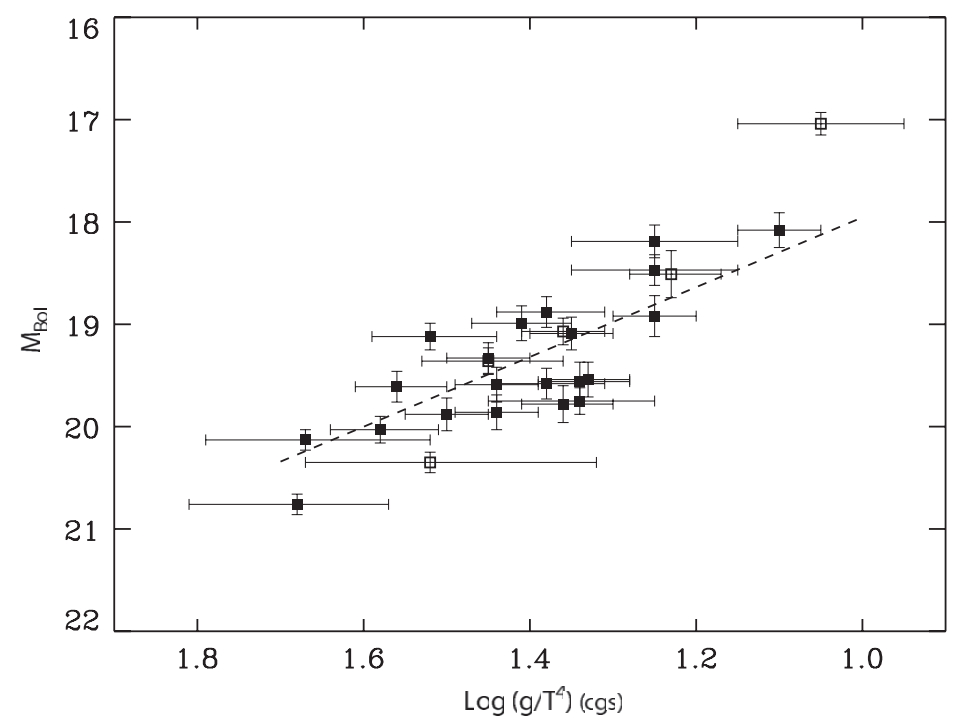

Figure 2. FGLR for blue supergiants in M81 (from Kudritzki et al. 2012). 


\section{Metallicities}

The effects of the chemical composition on Cepheid distances remains a controversial topic. Most empirical determinations are based on differential studies of Cepheids located at varying galactocentric distances in their host galaxy, since metallicity (obtained from HII regions) is known to decrease exponentially with radius in normal, isolated spiral galaxies. Variations in the period-luminosity relation with galactocentric distance can be interpreted as due to variations in the metallicity of the Cepheids (Kennicutt et al. 1998). This interpretation, however, has more recently led to somewhat paradoxical results, since the metallicity dependence, parameterized by $\gamma=\Delta \mu / Z$, has been found to be extremely high in a few well-studied galaxies, with $\gamma \simeq-0.6$ or smaller (see Gerke et al. 2011; and references therein). There are solid arguments against such a strong dependence (Majaess et al. 2011). On the other hand, these studies have also highlighted the need for improvements in the determinations of abundance gradients (see, for the maser host galaxy NGC 4258, Bresolin 2011).

Given the importance of deriving accurate metallicities in nearby galaxies to attain an improved calibration of stellar distance indicators, and more in general for the study of galactic chemical evolution, it is essential to be able to derive the metallicities of young galactic populations independently of the availability of HII region diagnostics, which remain the most widely used tool to investigate the chemical composition of star-forming galaxies. This is important to, among other things, study chemical abundance patterns that cannot be probed with nebular spectra and establish the correct zero point of the abundance scale. The fact that different calibrations of various nebular emission-line diagnostics can produce oxygen abundances of the parent ionized gas clouds that differ by as much as 0.6 dex is an unsolved problem of nebular astrophysics. As an example, Fig. 3 shows the galactocentric $\mathrm{O} / \mathrm{H}$ abundance gradient of the galaxy NGC 300, obtained by Bresolin et al. (2009) using the same set of 28 HII regions and emission-line fluxes, but applying different abundance diagnostics. Note the large systematic offsets among diagnostics, and the slight variation in the slope of the gradient.

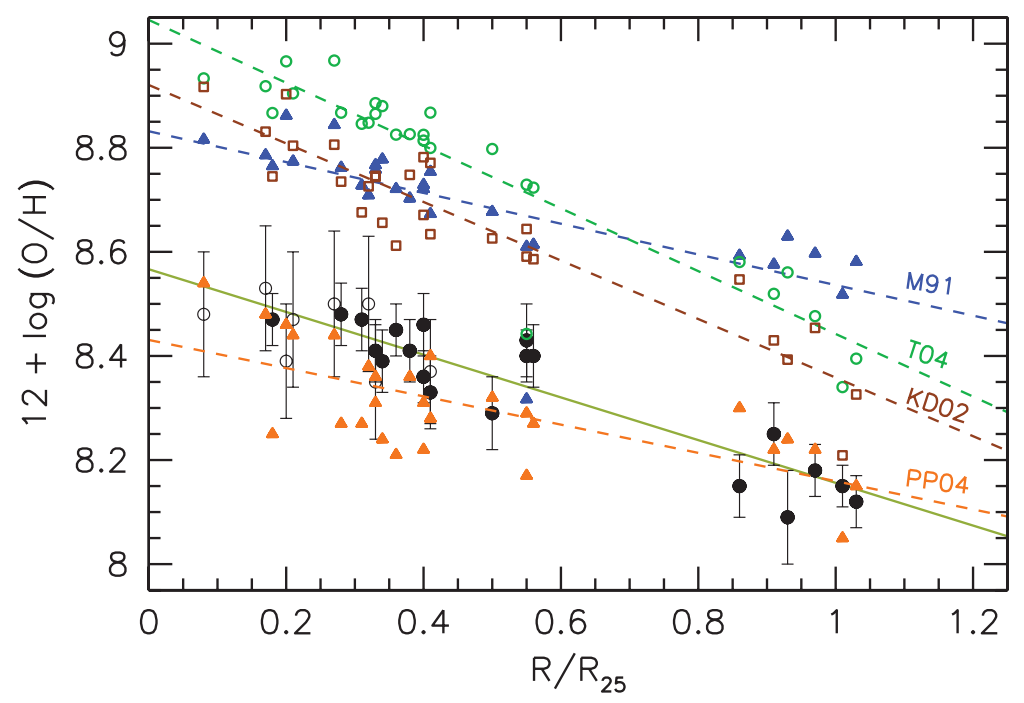

Figure 3. Galactocentric O/H abundance gradient of NGC 300, measured using the same set of 28 HiI regions, but applying five different abundance diagnostics, as shown by the different regression lines and symbols (from Bresolin et al. 2009). 
Our group has been thoroughly investigating the issue of the chemical composition of nearby galaxies by including both measurements of blue-supergiant metallicities (see, for details about how this is accomplished, Kudritzki et al. 2008, 2012) and of HiI regions through the 'direct' method. The latter involves the detection of faint auroral lines, such as $[\mathrm{OIII}] \lambda 4363$, that allow the derivation of the gas electron temperature, which strongly affects the line strengths. In Bresolin et al. (2009) we showed that the direct method produces nebular metallicities that are in excellent agreement with those from blue supergiant stars, at least for metallicities up to nearly the solar value (see Fig. 4). Even though the agreement reached in this particular case is impressive, analyses of other galaxies indicate that additional complications can arise. For example, some empirical evidence has been emerging lately indicating that for HiI regions around and above the solar metallicity the direct method might start to underestimate the true metallicity [see a recent discussion by Zurita \& Bresolin (2012) regarding the Andromeda galaxy, and the summary of nebular-versus-stellar results presented in the review by Stasińska et al. (2012)]. The reasons for this discrepancy are still elusive. Recent work by Nicholls et al. (2012) offers a possible explanation of the physical causes behind the effect, invoking non-Maxwellian distributions of electron energies. Investigations of metal-rich star-forming galaxies located within a few Mpc are crucial to shed light onto this issue from comparative abundance studies of young stars and HII regions.

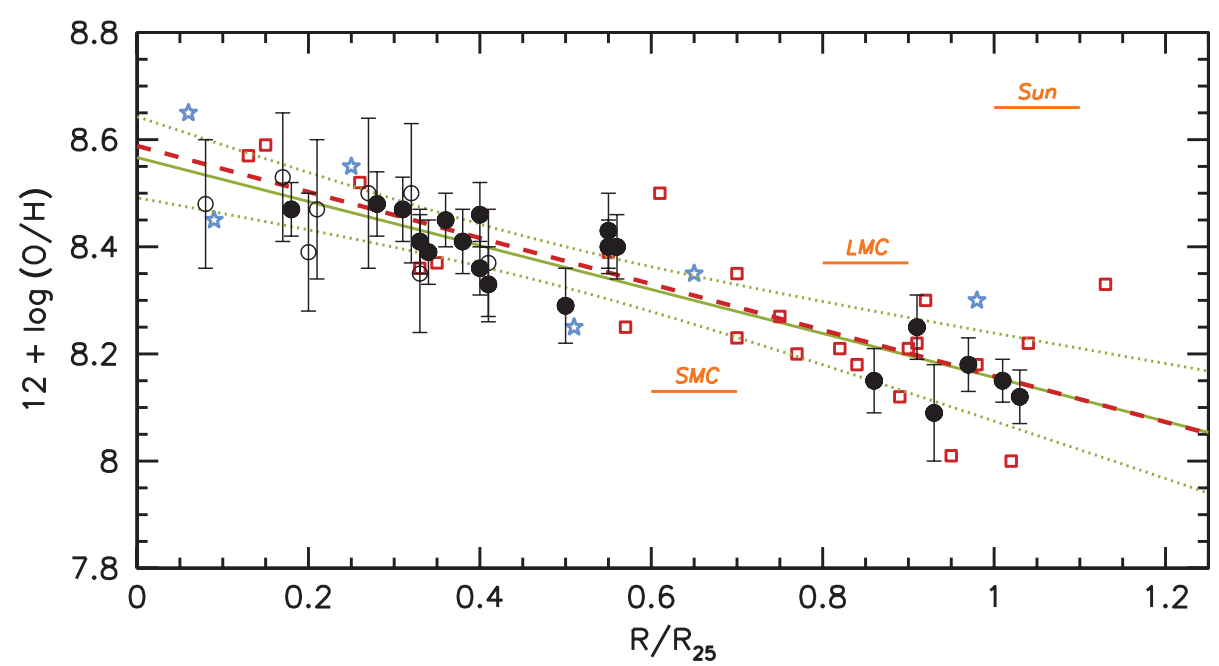

Figure 4. Galactocentric $\mathrm{O} / \mathrm{H}$ abundance gradient of NGC 300 obtained from the auroral-line method applied to HiI regions (solid symbols), compared with the blue-supergiant metallicities from Kudritzki et al. (2008; open squares: A supergiants, open stars: B supergiants. From Bresolin et al. 2009).

For these reasons, it is essential to continue to obtain metallicities of blue supergiants in nearby galaxies, which can be used not only to determine radial abundance gradients, but also to derive a mass-metallicity relationship for a restricted number of galaxies (see Fig. 5). Both of these allow us to test the nebular abundance diagnostics used ubiquitously in the literature for star-forming galaxies. 


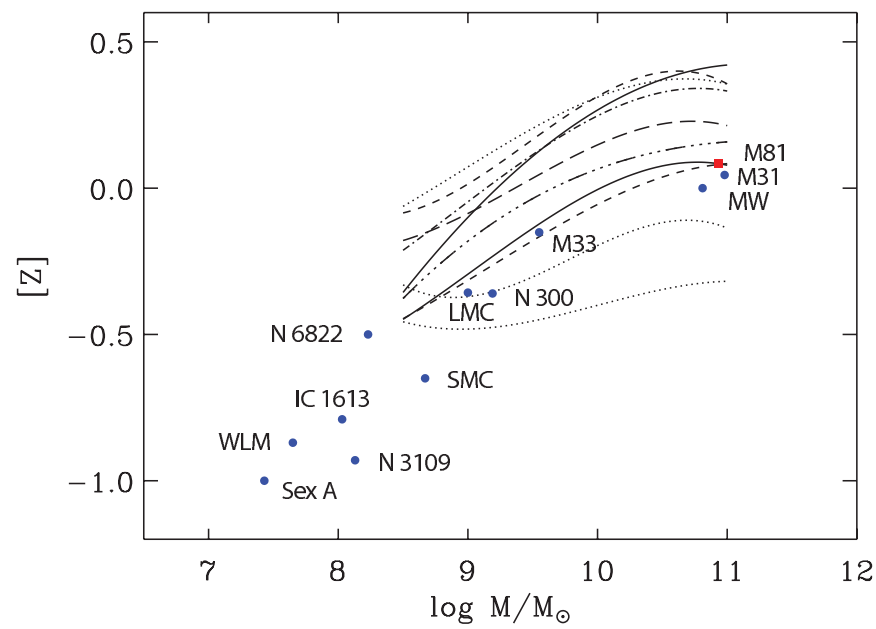

Figure 5. Galaxy mass-metallicity relation of SDSs galaxies obtained from various emission-line diagnostics (curves from Kewley \& Ellison 2008) and for a small number of nearby galaxies studied in detail by means of blue-supergiant spectroscopy (data points from Kudritzki et al. 2012).

\section{Acknowledgements}

We wish to express our gratitude to our collaborators on this project: Miguel Urbaneja, Norbert Przybilla, Wolfgang Gieren, Grzegorz Pietrzyński, Zach Gazak, and Vivian U.

\section{References}

Bono, G., Caputo, F., Fiorentino, G., et al. 2008, ApJ, 684, 102

Bresolin, F. 2011, ApJ, 729, 56

Bresolin, F., Gieren, W., Kudritzki, R.-P., et al. 2009, ApJ, 700, 309

Bresolin, F., Pietrzyński, G., Urbaneja, M. A., et al. 2006, ApJ, 648, 1007

Bresolin, F., Garnett, D. R., \& Kennicutt, R. C. 2004, ApJ, 615, 228

Freedman, W. L., Madore, B. F., Gibson, B. K., et al. 2001, ApJ, 553, 47

Gerke, J. R., Kochanek, C. S., Prieto, J. L., et al. 2011, ApJ, 743, 176

Kennicutt, R. C., Stetson, P. B., Saha, A., et al. 1998, ApJ, 498, 181

Kewley, L. J. \& Ellison, S. L. 2008, ApJ, 681, 1183

Kudritzki, R.-P. \& Urbaneja, M. A. 2012, ApSS, 341, 131

Kudritzki, R.-P., Urbaneja, M. A., Gazak, Z., et al. 2012, ApJ, 747, 15

Kudritzki, R.-P., Urbaneja, M. A., Bresolin, F., et al. 2008, ApJ, 681, 269

Kudritzki, R. P., Bresolin, F., \& Przybilla, N. 2003, ApJ, 582, L83

Macri, L. M., Stanek, K. Z., Bersier, D., et al. 2006, ApJ, 652, 1133

Majaess, D., Turner, D., \& Gieren, W. 2011, ApJ, 741 L36

Nicholls, D. C., Dopita, M. A., \& Sutherland, R. S. 2012, ApJ, 752, 148

Rizzi, L., Tully, R. B., Makarov, D., et al. 2007, ApJ, 661, 815

U, V., Urbaneja, M. A., Kudritzki, R.-P., et al. 2009, ApJ, 704, 1120

Urbaneja, M. A., Kudritzki, R.-P., Bresolin, F., et al. 2008, ApJ, 684, 118

Stasińska, G., Prantzos, N., Meynet, G., et al. 2012, Oxygen in the Universe, (EDP Sciences)

Suyu, S. H., et al. 2012, in: KIPAC workshop on the Hubble constant (arXiv:1202.4449)

Zurita, A. \& Bresolin, F. 2012, MNRAS, in press (arXiv:1209.1505) 